\title{
La ripisylve, un compartiment structurant des hydrosystèmes fluviaux intra-alpins et de piémonts
}

\author{
Hervé Piégay* \\ CNRS - UMR 5600 "Environnement - Ville - Société "
}

\section{I INTRODUCTION}

La ripisylve se définit comme un ensemble de groupements végétaux, la plupart étant dominés par une strate arborée, localisée sur la marge des cours d'eau et inféodée à des milieux régis par l'eau superficielle et souterraine. Alors qu'elle n'est développée que sur les berges des cours d'eau de petite taille, elle occupe l'ensemble du lit majeur des systèmes fluviaux les plus importants, ripi- signifiant la rive, la marge. Elle constitue dans ces cas là, une mosaïque végétale complexe (voir photo).

Longtemps peu considérée par les gestionnaires, elle apparaît aujourd'hui de plus en plus convoitée, certains allant jusqu'à la recréer sur certains tronçons. A partir de 6 exemples pris dans le bassin du Rhône, à savoir les basses vallées de l'Ain et de la Drôme et les moyennes vallées de l'Ubaye, du Giffre, de l'Ouvèze (Vaucluse) et de l'Ardèche (fig.1), nous montrerons le caractère récent de ces forêts pourtant considérées comme des formations primitives de la zone tempérée, l'influence du bois mort qui est introduit dans la rivière suite à leur développement, et enfin, les liens existant entre la ripisylve et la dynamique morpho-sédimentaire.

En s'appuyant sur les résultats acquis, des orientations de gestion seront formulées.

Comme l'ensemble de ces résultats est exposé succinctement sans que soient présentés les fondements bibliographiques et les méthodes utilisées, on pourra compléter son information à l'aide des références.

\section{II — DES FORMATIONS VÉGÉTALES RÉCENTES}

Les forêts alluviales sont des formations végétales récentes en France $[1,2]$. Alors qu'elles correspondent au climax stationnel des marges des rivières de la zone tempérée, rares sont celles qui existent encore au XVIII siècle. C'est ainsi que le cadastre napoléonien révèle que les lits majeurs de l'Ardèche et de l'Ouvèze étaient labourés jusqu'aux abords du lit mineur dans la première moitié du XIX ${ }^{\mathrm{e}}$ siècle. Si tous les systèmes fluviaux enregistrent un reboisement de leurs marges au cours de l'époque contemporaine, la chronologie de cette évolution diffère cependant d'un système à l'autre. Deux grands ensembles se distinguent : les cours d'eau des plaines intra-alpines et les cours d'eau de piémont.

* M. Piégay a obtenu le prix Henri Milon.

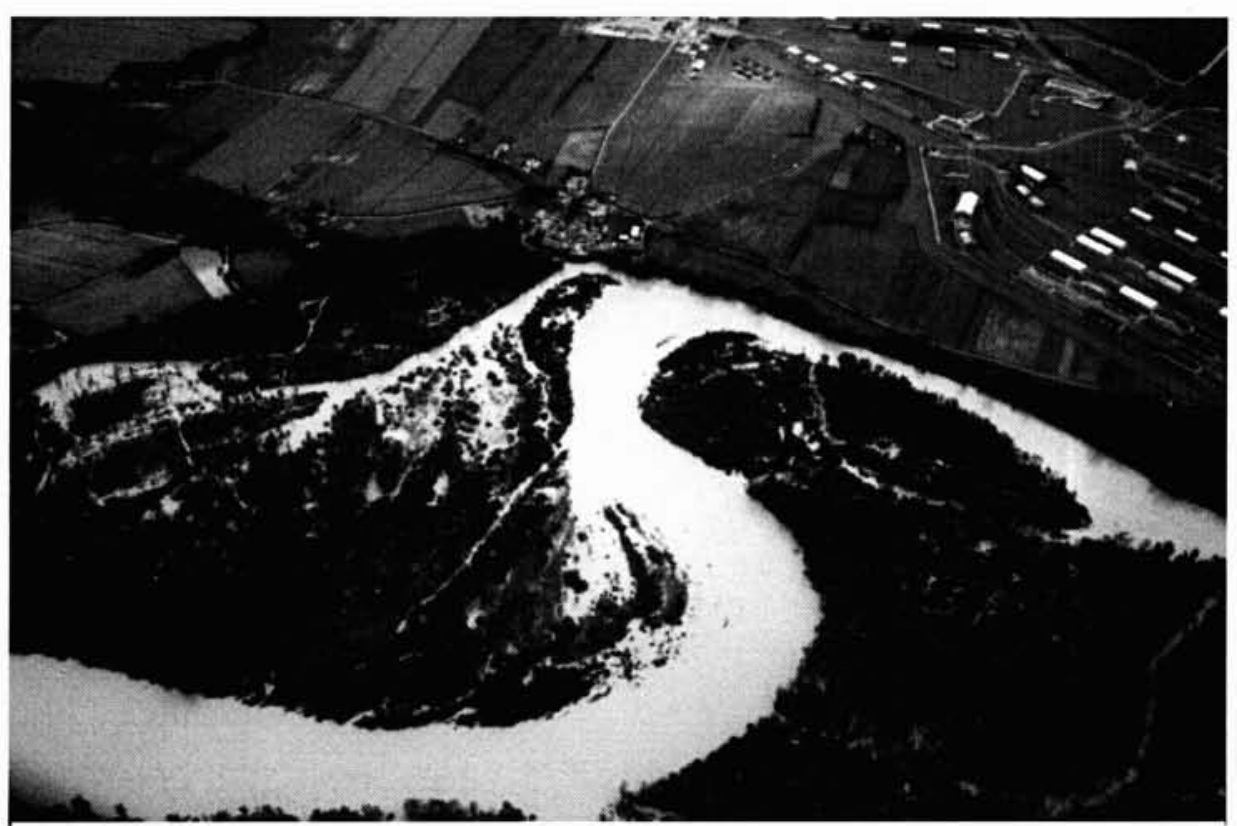

La mosaïque végétale de l'Ain, un cours d'eau à méandres mobiles. 


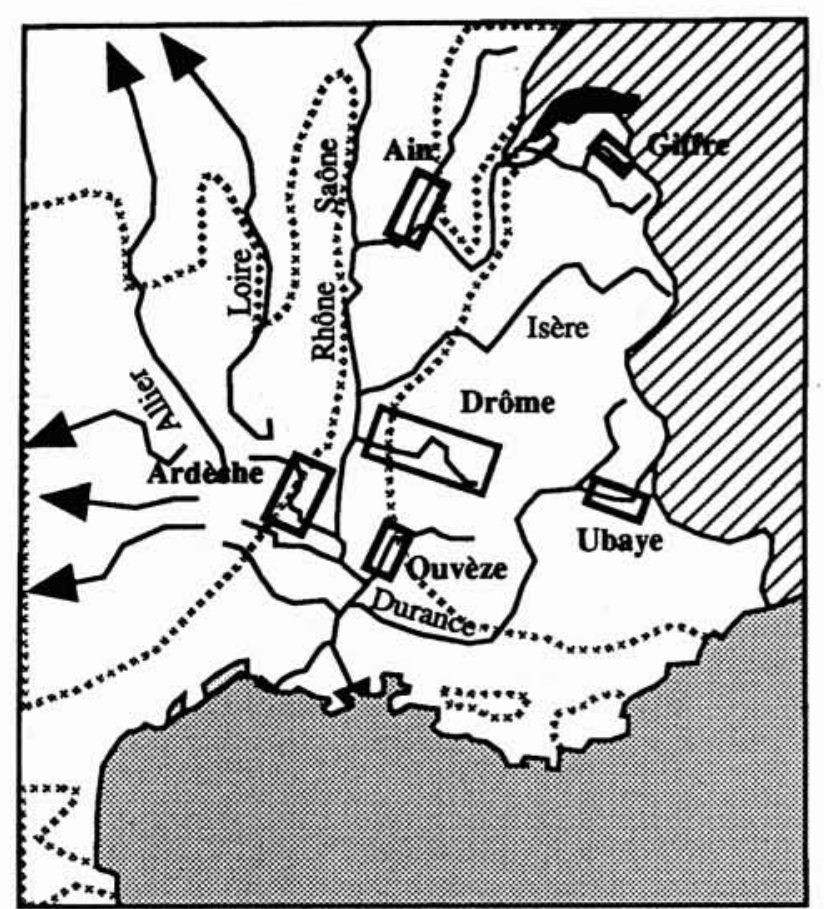

1. Localisation des tronçons étudiés.

Les premiers présentent une recolonisation végétale précoce. En 1733, l'espace riverain du Giffre, actuellement occupé par la forêt est déjà semi-naturel ; il est représenté sur la Mappe sarde comme une lande arbustive, une formation relativement ouverte. C'est en fait la politique conduite par les Sardes au XVIII siècle et qui imposait le maintien d'arbres en berges dans le but de limiter la divagation du lit, puis la politique forestière française de la fin du XIX siècle qui permit le développement de ce corridor boisé précoce et original à l'échelle de la France. Le cadastre français de la fin du XIXe siècle confirme ainsi l'existence d'une forêt riveraine, jardinée et dominée par les résineux sur toute la section moyenne du Giffre.

Les cours d'eau de piémont n'ont enregistré une recolonisation végétale de leur lit majeur qu'après 1945 (fig.2). Tel est le cas de l'Ain, de l'Ardèche et de l'Ouvèze. Cette tendance régionale pourrait être liée aux transformations qui ont touché le monde rural au cours des 30 Glorieuses ; l'agriculture qui associait traditionnellement culture et élevage et qui s'étendait sur l'ensemble des terroirs, y compris les moins productifs, comme les marges fluviales soumises à de fréquentes inondations, se spécialise et se reconcentre sur les meilleures terres. Parallèlement, le bois de chauffe est progressivement abandonné en tant que combustible domestique au profit du fuel. Ces deux évolutions synchrones expliquent ainsi l'abandon de ces marges par l'homme et leur évolution spontanée vers la forêt.

Néanmoins, la colonisation végétale ne touche pas seulement le lit majeur. Elle est également observée dans le lit mineur de toutes les rivières étudiées. Deux principales raisons, le plus souvent indissociables, expliquent ce phénomène :

- Le boisement du lit majeur observé sur les rivières de piémont s'est propagé en lit mineur. Les arbres qui se développent sur les marges du chenal influencent sa géométrie et modifient notamment sa rugosité ; les vitesses d'écoulement étant plus faibles en berge, elles permettent l'installation de jeunes arbres dans la bande active. Un tel ajustement des structures biologiques aux nouvelles contraintes hydrauliques s'arrête néanmoins au moment où un nouvel équilibre forêt chenal est trouvé. Tel est le cas de la plupart des rivières soumises à un développement arboré en 1945. Elles conservent en effet un lit d'une largeur constante après 1970 (fig.2). Ces observations viennent confirmer les travaux anglais qui ont montré que les cours d'eau intraforestiers à fond graveleux présentent un lit mineur d'une largeur inférieure et d'une profondeur supérieure à celui des cours d'eau drainant des corridors non boisés. La réduction de la largeur du lit mineur est ainsi synchrone de la colonisation arborée du lit majeur de l'Ain, de l'Ardèche ou de l'Ouvèze.

- Cette première raison ne permet pas d'expliquer la végétalisation du lit mineur de la Drôme ou de l'Ubaye, ces deux rivières présentant un lit majeur naturel et soumis aux débordements qui est peu étendu, voire inexistant sur des tronçons caractérisés par une végétalisation en lit mineur. Dans ce contexte, une telle végétalisation peut s'expliquer par une modification des écoulements à l'échelle de plusieurs décennies, voire d'un siècle. La colonisation arborée du lit majeur de l'Ubaye date ainsi des années 1910-1920, soit 30 à 40 ans seulement après le début du reboisement volontaire du bassin versant par les services RTM. La végétalisation de la Drôme est postérieure mais pourrait correspondre à une réduction :

- des pics de crue après 1925, comme l'atteste la chronique des maxima annuels enregistrée à la station de Luc-en-Diois,

- de la charge de fond à la suite du reboisement volontaire d'une partie du bassin et de la construction de plusieurs milliers de petits barrages sur les affluents les plus actifs.

\section{III $\square$ LA RIPISYLVE, UNE SOURCE DE DÉBRIS LIGNEUX}

La forêt alluviale alimente le système fluvial en débris ligneux et contribue en même temps à ralentir sa migration aval en le piégeant dans des sites préférentiels de dépôt $[3,4]$. C'est ainsi que sur près de $70 \mathrm{~km}$ de la rivière Drôme, les apports annuels de bois ont été estimés à près de 570 tonnes entre 1971 et 1991, 1400 tonnes étant actuellement stockées dans la bande active.

Les débris ligneux sont en fait présents sous différentes formes dans le système fluvial. Le tronc isolé se dépose préférentiellement dans la bande active, parallèlement à l'axe d'écoulement, les racines habituellement positionnées vers l'amont. L'embâcle, qui correspond à une accumulation hétérogène de troncs, de branches et de brindilles, est également une forme typique de dépôts dont la localisation est régie par le style fluvial. Sur les rivières à méandres, les embâcles sont préférentiellement présents sur les berges de concavités où ils peuvent former dans certains cas une ligne de débris jointifs et perméables. $75 \%$ des masses de bois localisées sur un site de 10 ha sur l'Ardèche, soit 371 tonnes de bois, sont disposées au contact de la forêt et du chenal. Dans les rivières à tresses, les embâcles se déposent plutôt sur la partie supérieure des bancs de galets médians ou au contact d'arbustes.

L'étude conduite dans les concavités de trois méandres de l'Ain a montré que la distribution des masses de bois est très variable au sein de chaque concavité et d'une concavité à l'autre [5] (fig.3). La position des embâcles en berges varie 


\section{Largeur de la ripisylve 1947-91}

Transects dont l'augmentation de la largeur forestière est :

suptrieure $\mathbf{1} 100 \mathrm{~m}$

comprise entre $50 \mathrm{et} 100 \mathrm{~m}$

- comprise entre 20 et moins de $50 \mathrm{~m}$

Transects dont la réduction de la largeur forestière est :

suptrieure \& $100 \mathrm{~m}$.

comprise entre 50 et $100 \mathrm{~m}$.

w comprise entre 20 et moins de $50 \mathrm{~m}$.

Transects dont la régénération de la bande active est :

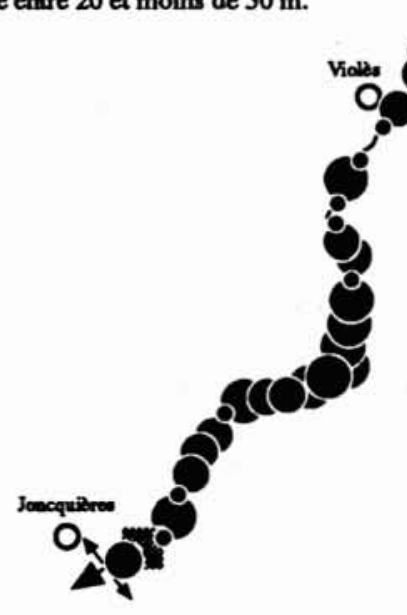

suptrieure $\$ 50 \mathrm{~m}$

- comprise entre 20 et $50 \mathrm{~m}$

Transects dont la colonisation végétale est :

supérieure $\mathbf{1 0 0 \mathrm { m }}$

comprise entre 50 et $100 \mathrm{~m}$

comprise entre 20 et moins de $50 \mathrm{~m}$
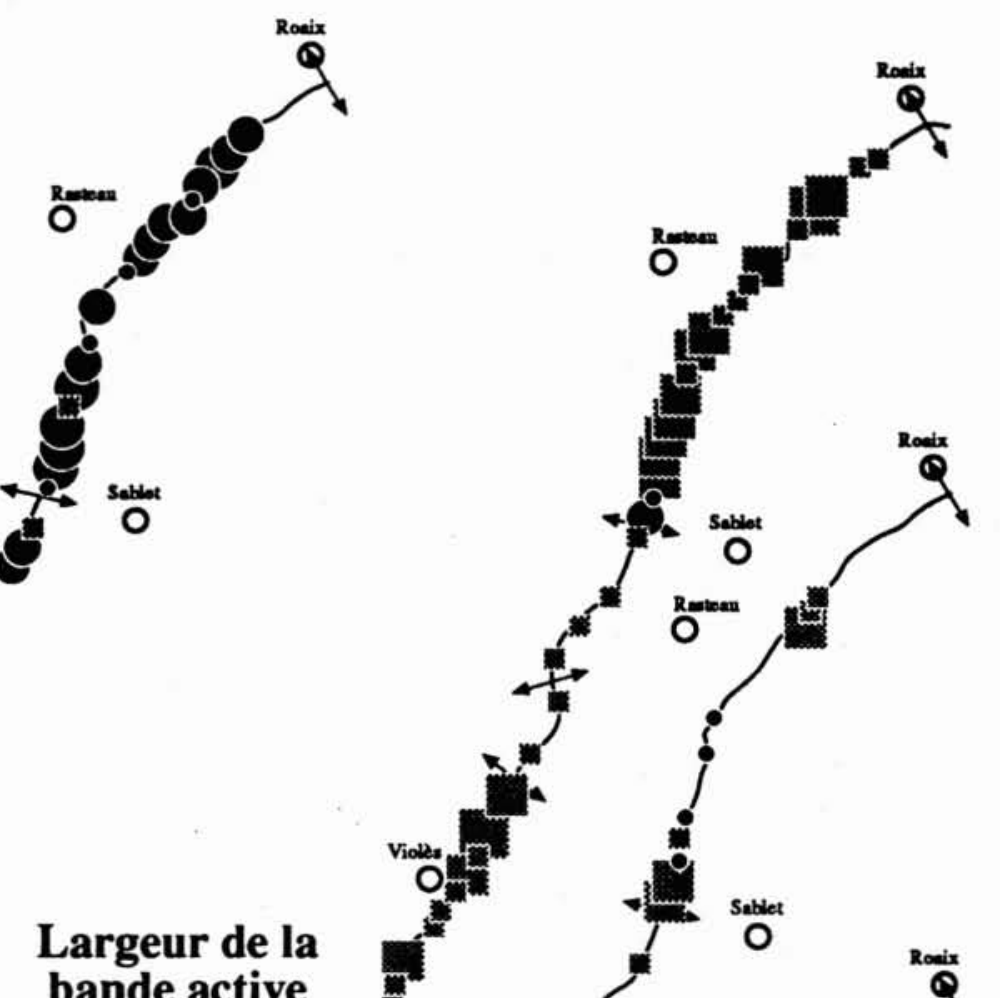

Largeur de la
bande active

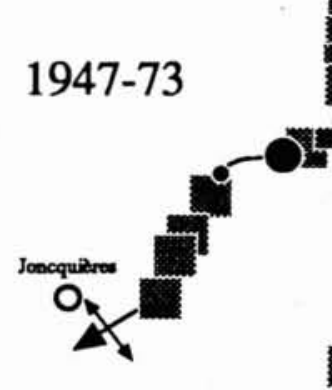

1973-91
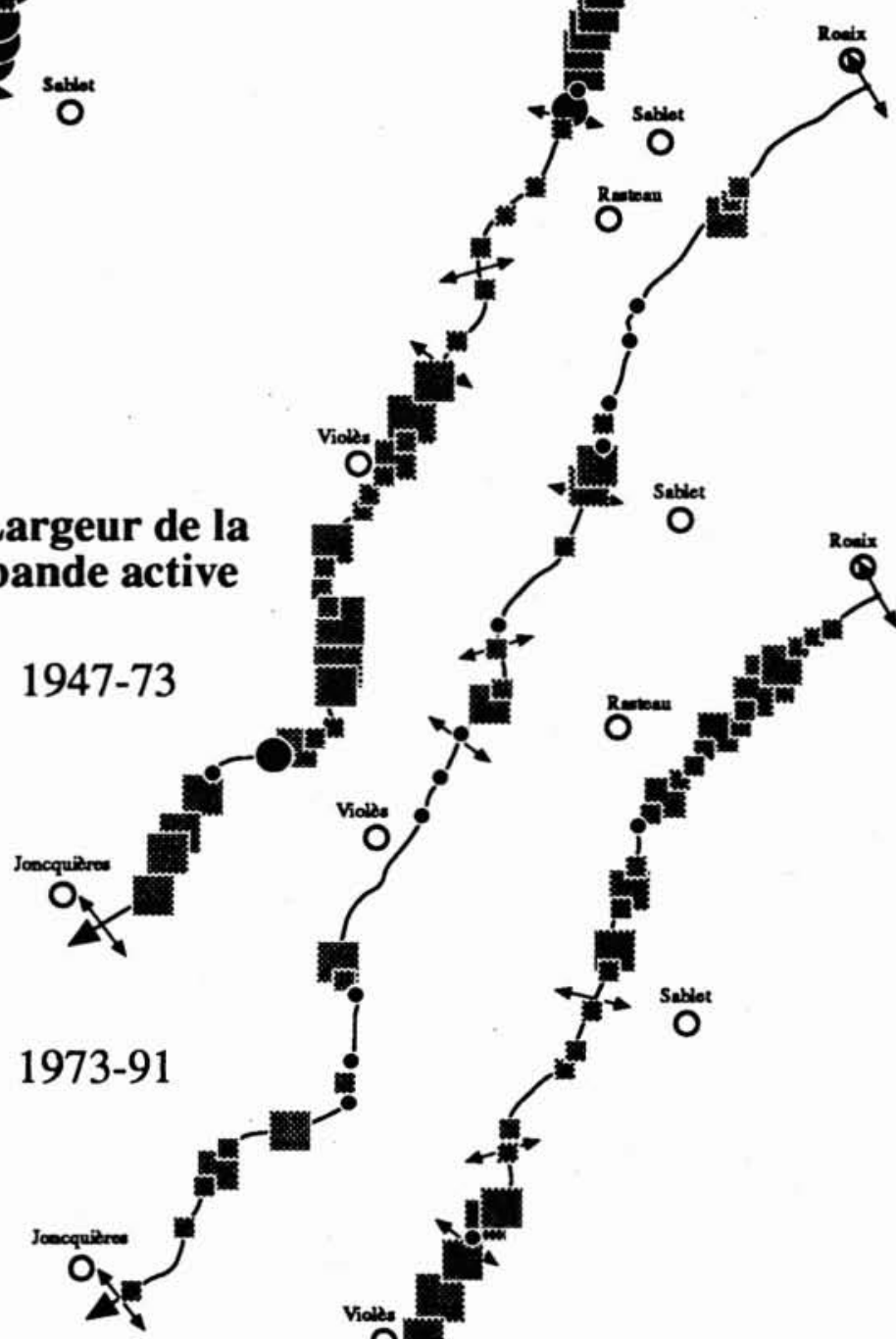

Routam

0

Retert
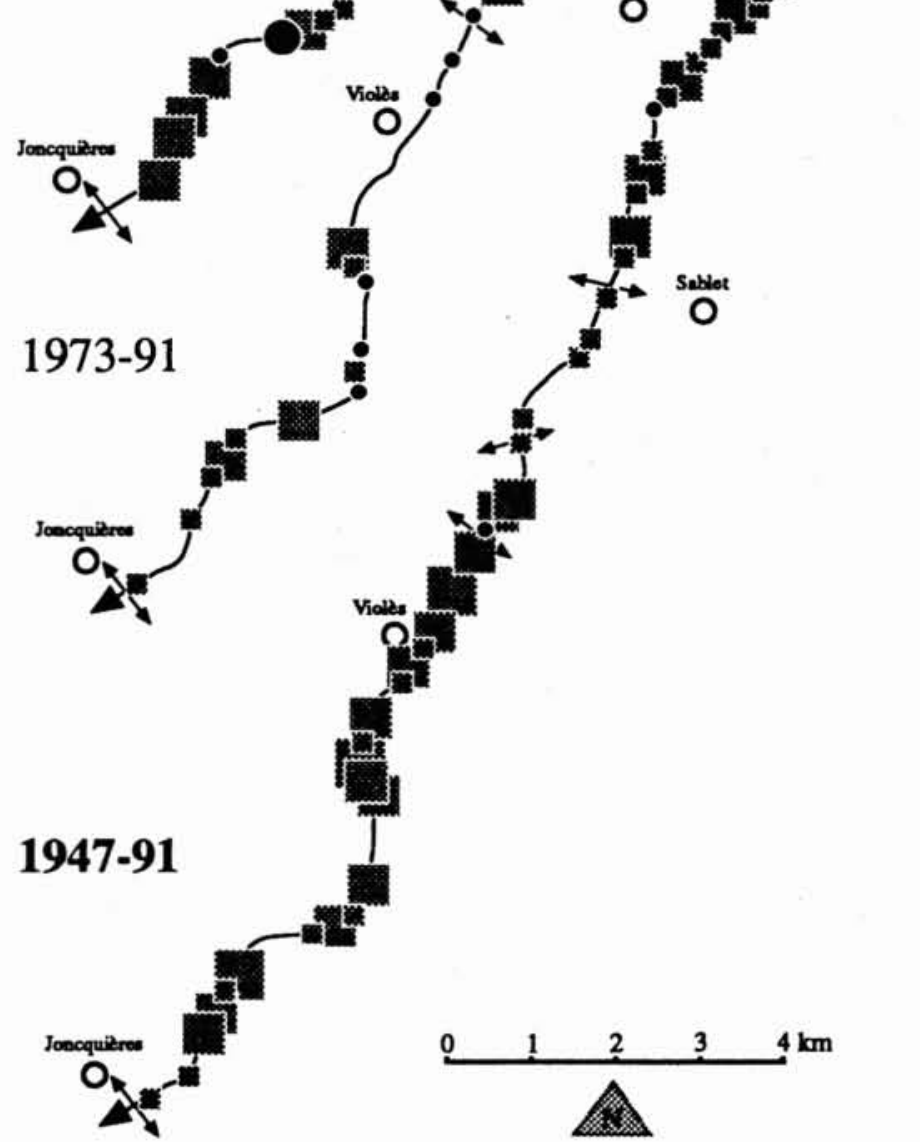

2. La recolonisation végétale de l'Ouvèze entre 1947 et 1991 - cas du tronçon de Roaix à Joncquière. 

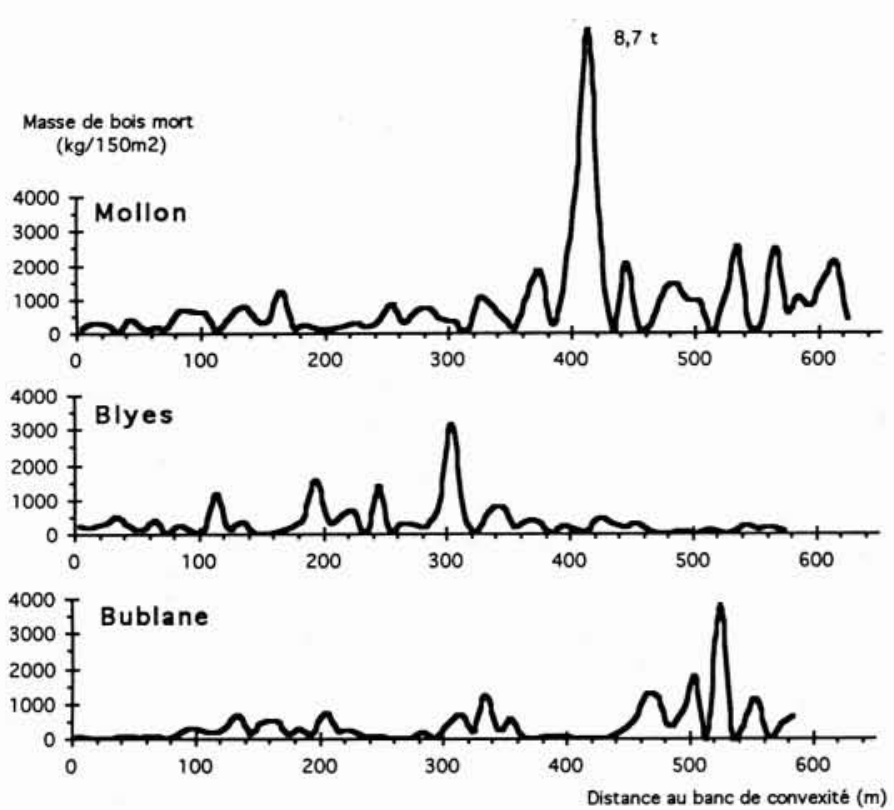

3. Distribution des masses de bois sur trois concavités de l'Ain : Mollon, Blyes et Bublane.

longitudinalement. Les masses de bois déposées par les crues sont d'autant plus importantes que la zone d'observation présente une berge basse ou qu'elle est localisée au droit du chenal principal. L'équation ci-après permet d'estimer la masse de bois (en t.ha-1), Y, en fonction de la hauteur de berge $\left(\mathrm{X}_{1}\right)(\mathrm{m})$ et de l'angle d'impact des écoulements (plus cet angle est ouvert, plus la parcelle d'observation est localisée au droit du chenal principal) $\left(\mathrm{X}_{2}\right.$ - degré) :

$$
\begin{aligned}
& \log (Y)=-0,403 X_{1}+0,014 X_{2}+3,05 \\
& \left(r^{2}=0.6 ; p<0.0001\right)
\end{aligned}
$$

Dans les secteurs moins fournis en bois, où seules sont présentes des branches et des brindilles remobilisées par les crues, la nature du couvert végétal intervient pour expliquer la distribution des masses de bois. Dans la concavité de Blyes, les stations caractérisées par une forte densité d'arbres disposent ainsi d'une masse de débris ligneux plus importante que les stations qui présentent une moindre densité d'arbres.

Au sein des concavités, le bois mort est d'abord présent en berge, les masses déposées décroissant progressivement du chenal vers l'intérieur du lit majeur boisé. Trois équations calculées à partir des données collectées sur la station d'observation de Mollon (Ain) mettent en lumière une tendance linéaire, celle-ci étant variable d'une année à l'autre ; $\mathrm{Y}$ est la surface occupée par les embâcles $\left(\mathrm{m}^{2} / \mathrm{m}\right)$ et $\mathrm{X}$ la distance entre le chenal et le point d'observation $(\mathrm{m})$ :

$$
\begin{aligned}
& Y_{1903}=-0,0006 X+0,10 \\
& Y_{1904}=-0,0008 X+0,12 \\
& Y_{1905}=-0,0014 X+0,12
\end{aligned}
$$

Les masses de bois déposées dans les concavités varient également d'un site à l'autre. L'abondance du bois dépend d'une part de la capacité de la géoforme à retenir les débris plus le méandre est formé et plus les berges sont basses, plus le site est propice au dépôt - et d'autre part de la capacité d'alimentation du tronçon - le bois mort est d'autant plus abondant au sein d'une berge de concavité que le tronçon amont présente des berges érodées boisées.

Les rivières en tresses présentent une capacité de stockage qui est moindre que celle des rivières à méandres et très variable d'un tronçon à l'autre (fig.4). Sur la Drôme, la bande active abrite ainsi en moyenne une masse de bois de l'ordre de 2,2 t.ha-1. Dans les sites préférentiels, la masse peut atteindre 35 t.ha $^{-1}$ contre 90 tha $^{-1}$ sur l'Ain dans les zones de concavités les plus riches. Quatre principaux facteurs semblent régir la capacité de stockage de la bande active comme le souligne l'équation suivante où $\mathrm{Y}$ est le nombre d'embâcles par sections de $500 \mathrm{~m}$ de bande active, $X_{1}$. l'indice de tressage, $X_{2}$, la pente du chenal $(\mathrm{m} / \mathrm{m}), X_{3}$, le nombre de contacts entre la bande active et les zones boisées et $\mathrm{X}_{4}$, le nombre de sections de $500 \mathrm{~m}$ identifiées comme ayant une ou deux berges érodées boisées et localisées sur $2 \mathrm{~km}$ à l'amont du site de mesure (modalités de 0 à 8 ).

$$
\begin{gathered}
Y=14.2 X_{1}-1124.08 X_{2}+7.5 X_{3}+10.19 X_{4}-40.34 \\
\left(r^{2}=0.42 ; p<0.0001\right)
\end{gathered}
$$

L'abondance du bois mort dépend ainsi de la géométrie du lit. Les dépôts sont importants lorsque le lit présente des chenaux multiples et des bancs de galets caractérisés par une faible épaisseur de la nappe d'eau au moment des hautes eaux, une forte interpénétration chenal-unités végétalisées, une faible pente locale. Malgré tout, la présence du bois est également dépendante, comme dans les systèmes à méandres, de la disponibilité locale du bois. Une section est d'autant plus fournie en bois que les sections immédiatement amont sont soumises à une forte érosion de leurs berges boisées.

\section{IV — LA RIPISYLVE, UN FREIN À L'ÉCOULEMENT}

La forêt alluviale ralentit fortement les écoulements de débordement comme le souligne l'analyse de l'altitude des amas de brindilles déposés sur les troncs et branches maîtresses au moment du pic de crue [6]. Ces indicateurs, utilisés lors de l'étude des crues qui ont touché l'Ain, l'Ardèche et l'Ouvèze en 1992, ont permis de montrer l'existence d'écoulements en lit majeur, perpendiculaires au chenal principal. Une pente de $2,2 \%$, soit $65 \mathrm{~cm}$ de dénivelé sur une distance de $30 \mathrm{~m}$, a ainsi été mesurée sur une marge de la rivière Ouvèze, cette zone de contact ripisylve-lit mineur étant obturée lors de la crue du 22 septembre 1992 par une ligne de débris ligneux. Dans des secteurs de moindre rugosité, des pentes transversales de $0,8 \%$ et $1,3 \%$ ont également été relevées sur les marges de l'Ardèche (crue décennale) et de l'Ouvèze (crue plus que centenale).

Ce schéma est néanmoins plus complexe lorsque le lit majeur abrite d'anciens chenaux partiellement végétalisés. En effet, ceux-ci drainent une partie des écoulements de débordement, une pente transversale de la ligne d'eau étant une fois encore observée sur leurs marges.

La forêt constitue également un filtre sédimentaire efficace $[7,8]$. La taille granulométrique et l'épaisseur des sédiments fins déposés en lit majeur sont à la fois dépendants de l'altitude et de la position géographique des points d'échan- 


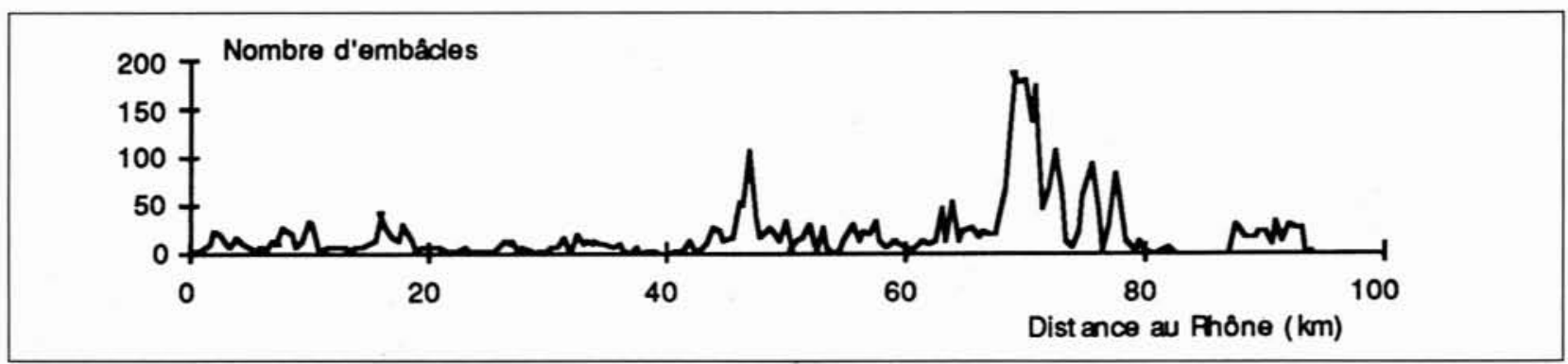

4. Répartition aval-amont du nombre d'embâcles dans le lit de la Drôme.

tillonnage. Plus ils sont hauts ou éloignés de l'axe principal, plus ils reçoivent une sédimentation fine et peu épaisse. Les zones hautes sont en effet soumises à des lames d'eau moins épaisses et de moindre compétence ; de même, plus le point de prélèvement est éloigné du bras principal, plus la zone de contact entre ce point et ce bras filtre les sédiments entrant dans le lit majeur. Au delà d'une certaine distance, l'épaisseur des sédiments déposés lors de chaque crue n'excède pas un millimètre. L'altitude et la distance ne constituent néanmoins pas les seuls facteurs qui régissent la distribution des sédiments dans le lit majeur, la densité de la végétation joue également un rôle-clé. Les unités arborées pionnières composées de jeunes plants (4 000-6 000 individus par hectare) ont une plus grande aptitude à piéger les sédiments que les unités matures (300-500 individus par hectare). Sur l'Ain, les unités boisées de moins de 10 ans ont en moyenne un taux de sédimentation de 2,3 à $3,6 \mathrm{~cm} / \mathrm{an}$ contre 1,7 à $2,03 \mathrm{~cm} /$ an dans les unités qui ont 50 ans. Les équations suivantes où $\mathrm{Y}$ est l'épaisseur totale des sédiments fins de lit majeur $(\mathrm{cm})$ et $\mathrm{X}$ est la durée durant laquelle la surface est occupée par de la végétation (année) permettent de modéliser cette dynamique sur la rivière d'Ain :

Site 1: $Y=1,64 X+19,7\left(r^{2}=0,49\right)$

Site $2: \mathrm{Y}=1,55 \mathrm{X}+8,0\left(\mathrm{r}^{2}=0,53\right)$
L'analyse de la sédimentation sur les marges de l'Ouvèze à la suite de la crue de septembre 1992 est également riche d'enseignement. Deux démarches ont été conduites. La première repose sur la comparaison de deux sites de l'Ouvèze, l'un étant boisé (Station a), l'autre étant occupé par de la vigne (Station b). Comme le soulignent les trois équations ci-après, la rédution de la médiane des sédiments s'effectue sur une distance beaucoup plus courte sous forêt que dans une parcelle viticole. Si un lien existe en effet entre la médiane des sédiments déposés lors de la crue de septembre $1992(\mathrm{Y}-\mu)$ et la distance du point de prélèvement au chenal $(X-\mu)$, ce lien diffère néanmoins selon la nature de l'occupation de la station :

Station a / rive gauche : $Y=-0,005 X+1,5 \quad\left(r^{2}=0,67\right)$
Station a / rive droite : $Y=-0,0085 X+2,4 \quad\left(r^{2}=0,5\right)$

Station b : $\log (\mathrm{Y})=-0,0011 \mathrm{X}+1,9\left(\mathrm{r}^{2}=0,53\right)$

La seconde démarche conduite sur un site naturel de la rivière repose sur une analyse diachronique (fig.5) : la médiane des sédiments de surface, déposés par la dernière crue, est comparée à celle des sédiments déposés antérieurement, un prélévement étant effectué tous les $15 \mathrm{~cm}$ dans les

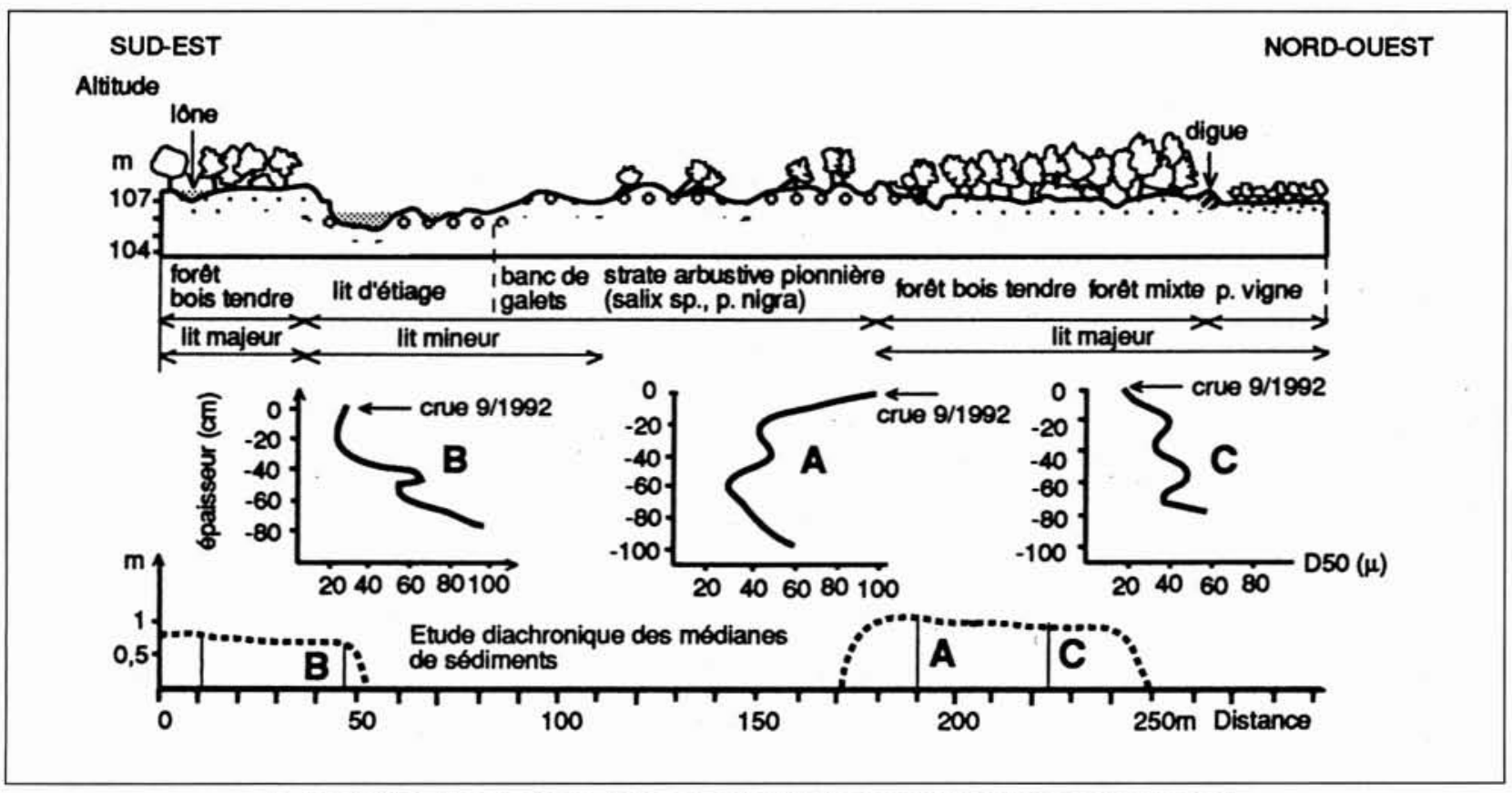

5. Evolution diachronique de la granulométrie des sédiments de débordement dans le lit majeur de l'Ouvèze - site de Violès. 
trois carottes étudiées. Il apparait que le sédiment déposé par la dernière crue est beaucoup plus grossier que celui déposé antérieurement sur le point d'observation A localisé sous forêt à $6 \mathrm{~m}$ de la bande active. En revanche, le sédiment déposé par la dernière crue aux points observation $\mathrm{B}$ et $\mathrm{C}$, localisés respectivement à 33 et $55 \mathrm{~m}$ de la bande active, s'inscrit dans la tendance historique ; il est globalement plus fin que les dépôts précédents. Ces résultats permettent d'affirmer qu'au delà de $40 \mathrm{~m}$ sous forêt, la crue du 22 septembre 1992 sur l'Ouvèze, considérée comme plus que centenale, n'est pas un événement granulométriquement exceptionnel.

\section{$\mathrm{V}$ @ CONCLUSION : QUELQUES PRINCIPES DE GESTION}

Trois grands principes de gestion doivent être retenus à l'issue de ces résultats :

- La ripisylve est un compartiment récent des hydrosystèmes fluviaux en France à l'origine de changements morphologiques, notamment un rétrécissement et un approfondissement du lit, et de nouveaux processus biomorphologiques consécutifs à l'introduction du bois mort dans la rivière. C'est pourquoi, tout principe de gestion ne peut se fonder sur un état initial ou de référence : à système nouveau, gestion nouvelle [9]. La présence d'une forêt en lit majeur est un élément à la fois positif et négatif pour les collectivités riveraines (productivité sylvicole, attraction paysagère, autoépuration, risques induits lors des crues...). Selon le tronçon considéré, le gestionnaire devra trouver un juste équilibre entre l'optimum écologique et la satisfaction des usages. Maintenue ici pour des raisons écologiques ou de gestion des débordements, la forêt pourra être réduite là, notamment dans certains lits mineurs compte tenu des risques d'inondation nouveaux liés à la réduction de la capacité d'écoulement. Une politique simpliste privilégiant le " tout restaurer" ou le " tout entretenir" ne peut pas se concevoir, la question de fond étant de savoir ce que l'on souhaite faire de tel ou tel tronçon.

- L'entretien de la végétation riveraine et des embâcles doit être conduit en tenant compte d'un certain nombre de règles $[10,11]$. Les orientations à formuler en matière d'entretien se fondent non seulement sur les résultats développés précédemment mais également sur de très nombreuses références scientifiques, qui mettent en lumière le rôle écologique du bois mort dans les systèmes fluviaux de la zone tempérée [12]. Par ailleurs, compte tenu de la taille du réseau hydrographique français, il apparaît qu'il n'est financièrement pas possible de l'entretenir dans sa totalité, des priorités doivent donc être définies. C'est ainsi que les collectivités locales qui prennent en charge l'entretien de bassins de 1000 à $2000 \mathrm{~km}^{2}$ devraient en priorité axer leurs interventions sur le réseau secondaire et non pas sur l'axe principal caractérisé par un risque moindre de formation de bouchon ligneux, le lit et les sections de ponts étant suffisamment larges pour évacuer des débris par ailleurs de petite taille. Les débris ligneux déposés par exemple dans le lit de la Drôme sont avant tout des arbustes flexibles peu à même de former des bouchons organiques. L'entretien devrait en fait être graduel, varier en intensité selon les caractéristiques des tronçons, certains pouvant ainsi ne pas être entretenus.

- La forêt filtre les écoulements de débordement et constitue ainsi un espace tampon naturel entre un système fluvial caractérisé par des crues violentes et un lit majeur large et cultivé. Elle constitue donc un filtre sédimentaire et organique et ralentit les écoulements qui sont alors de moindre compétence dans un lit majeur cultivé. Le maintien d'un corridor boisé continu d'une largeur d'au moins $30 \mathrm{~m}$ est ainsi à préconiser sur les rivières de piémont et notamment les affluents de rive gauche du Rhône, à savoir la Drôme, l'Ouvèze, ou l'Eygues. Si la préservation du corridor existant est une nécessité à la fois écologique et économique, sa restauration doit également être envisagée. Ce corridor est en effet soumis depuis les dernières décennies à une forte pression foncière, agricole ou touristique, et est en cours de morcellement; il ne joue donc plus totalement son rôle de protection. Les principaux dommages subis par les cultures dans le lit majeur de l'Ouvèze en 1992 ont ainsi été enregistrés dans des secteurs sur lesquels le corridor boisé était étroit ou inexistant. C'est pourquoi des réflexions sont actuellement en cours, en concertation avec le Conseil Général de Vaucluse, pour engager une restauration de la ripisylve sur des sites de l'Ouvèze, du Calavon ou de la Durance.

\section{Références}

[1] Bravard J.-P. ET Dupont P. (1994), «La dynamique végétale et les crues sur la marge des cours d'eau, Colloque de la Société Hydrotechnique de France, 23èmes Journées de l'hydraulique sur le thème "Crues et inondations", Nîmes, 14 et 16 septembre 1994, 277-289.

[2] 1996, «La forêt d'inondation des rivières à forte énergie, un patrimoine écologique à gérer». Annales de Géographie, 590 : 347-368.

[3] 1993, «Nature, mass and preferential sites of coarse woody debris deposits in the lower Ain valley (Mollon reach), Francew. Regulated Rivers Res. Manag., 8 : 359-372.

[4] Gurnell. A. M., sous presse, «Coarse woody debris and river geomorphological style : examples from european rivers», Geomorphology.

[5] 1995, «Dynamiques et gestion de la forêt alluviale de cinq cours d'eau à charge grossière du bassin du Rhône : l'Ain, l'Ardèche, le Giffre, l'Ouvèze et l'Ubaye (XIXe-XXe siècles)», Thèse de géographie (Université Paris IV) - Sorbonne, 529 pp.

[6] Sous presse, «Interactions between floodplain forest and overbank flows : data from three piedmont rivers of southeastern France», Global ecology and biogeography letters.

[7] 1995, «Dynamiques et gestion de la forêt riveraine de cinq cours d'eau à charge grossière du bassin du Rhône», Bull. de la Société Langudocienne de Géographie : la maîtrise de l'eau en Languedoc, 59 (1-2) : 63-81

[8] BRAVARD J.P., sous presse, $\alpha$ The reactions of a mediterranean riparian forest to a major hydrological event, the 1 in 400 year flood (22.09.1992) in the Ouvèze river. Drôme-Vaucluse, France», Earth Surface, Processes and Landforms.

[9] BRAVARD J.-P. ET Dupont P., 1994, «The french water Law : a new approach for alluvial hydrosystem management, French alpin and perialpin stream exemples», in : R.A. Marston et Hasfurther V. R., Annual summer symposium of the American Water Resources Association, effects of humaninduced changes on hydrologic systems, American Water Resources Association, Jackson Hole, Wyoming, USA, 371-383.

[10] 1994, «Quelques éléments de réflexion pour une gestion équilibrée des marges boisées des cours d'eau de plaines alluviales», rapport, CEMAGREF - Division Biologie des Ecosystèmes Aquatiques, Lyon, $130 \mathrm{p}$.

[11] MARIdet L., GILARD O. et Thévenet A., 1996, «L'embâcle de bois en rivière, un bienfait écologique ou un facteur de risques naturels ?», La Houille Blanche, 5 : 32-38.

[12] MARIDET L., 1994, «Formations végétales arborées riveraines des cours d'eau et potentialités piscicoles (revue bibliogra- 